\title{
Generalized Contractive Set-Valued Maps on Complete Preordered Quasi-Metric Spaces
}

\author{
J. Marín, S. Romaguera, and P. Tirado \\ Instituto Universitario de Matemática Pura y Aplicada, Universitat Politècnica de València, Camí de Vera s/n, 46022 València, Spain \\ Correspondence should be addressed to S. Romaguera; sromague@mat.upv.es
}

Received 12 October 2012; Accepted 8 December 2012

Academic Editor: Gerald Beer

Copyright (C) 2013 J. Marín et al. This is an open access article distributed under the Creative Commons Attribution License, which permits unrestricted use, distribution, and reproduction in any medium, provided the original work is properly cited.

By using a suitable modification of the notion of a $w$-distance we obtain some fixed point results for generalized contractive setvalued maps on complete preordered quasi-metric spaces. We also show that several distinguished examples of non-metrizable quasi-metric spaces and of cones of asymmetric normed spaces admit $w$-distances of this type. Our results extend and generalize some well-known fixed point theorems.

\section{Introduction and Preliminaries}

Throughout this paper the letters $\mathbb{R}, \mathbb{R}^{+}, \mathbb{N}$, and $\omega$ will denote the set of real numbers, the set of non-negative real numbers, the set of positive integer numbers and the set of non-negative integer numbers, respectively. Our basic references for quasimetric spaces are $[1,2]$ and for asymmetric normed space it is [3].

A quasi-pseudometric on a set $X$ is a function $d: X \times$ $X \rightarrow \mathbb{R}^{+}$such that for all $x, y, z \in X:(\mathrm{i}) d(x, x)=0$; (ii) $d(x, y) \leq d(x, z)+d(z, y)$.

If $d$ satisfies conditions (i) and (ii) above but we allow $d(x, y)=+\infty$, then $d$ is said to be an extended quasi-pseudometric on $X$.

Following the modern terminology, a quasi-pseudometric $d$ on $X$ satisfying $\left(\mathrm{i}^{\prime}\right) d(x, y)=d(y, x)=0$ if and only if $x=y$ is called a quasi-metric on $X$.

If the quasi-metric $d$ satisfies the stronger condition $\left(\mathrm{i}^{\prime \prime}\right)$ $d(x, y)=0$ if and only if $x=y$, we say that $d$ is a $T_{1}$ quasimetric on $X$.

A $\left(T_{1}\right)$ quasi-metric space is a pair $(X, d)$ such that $X$ is a nonempty set and $d$ is a $\left(T_{1}\right)$ quasi-metric on $X$.

Each extended quasi-pseudometric $d$ on a set $X$ induces a topology $\tau_{d}$ on $X$ which has as a base the family of open balls $\left\{B_{d}(x, \varepsilon): x \in X, \varepsilon>0\right\}$, where $B_{d}(x, \varepsilon)=\{y \in X: d(x, y)<$ $\varepsilon\}$ for all $x \in X$ and $\varepsilon>0$.

The closure with respect to $\tau_{d}$ of a subset $A$ of $X$ will be denoted by $\mathrm{cl}_{\tau_{d}} A$.
Note that if $d$ is quasi-metric then $\tau_{d}$ is a $T_{0}$ topology, and if $d$ a $T_{1}$ quasi-metric then $\tau_{d}$ is a $T_{1}$ topology on $X$.

Given a quasi-metric $d$ on $X$, the function $d^{-1}$ defined by $d^{-1}(x, y)=d(y, x)$ for all $x, y \in X$, is also a quasimetric on $X$, and the function $d^{s}$ defined by $d^{s}(x, y)=$ $\max \{d(x, y), d(y, x)\}$ for all $x, y \in X$, is a metric on $X$.

There exist several different notions of Cauchyness and quasi-metric completeness in the literature (see, e.g., [2]). In our context will be useful the following general notion.

Definition 1. A quasi-metric $d$ on a set $X$ will be called complete if every Cauchy sequence $\left(x_{n}\right)_{n \in \omega}$ in $(X, d)$ converges with respect to the topology $\tau_{d^{-1}}$ (i.e., there exists $z \in X$ such that $\left.\lim _{n} d\left(x_{n}, z\right)=0\right)$, where the sequence $\left(x_{n}\right)_{n \in \omega}$ is said to be Cauchy if for each $\varepsilon>0$ there exists $n_{0} \in \mathbb{N}$ such that $d\left(x_{n}, x_{m}\right)<\varepsilon$ whenever $n_{0} \leq n \leq m$. If $d$ is complete we will say that the quasi-metric space $(X, d)$ is complete.

Kada et al. introduced in [4] the notion of $w$-distance for metric spaces and obtained, among other results, $w$-distance versions of the celebrated Ekeland variational principle [5] and the nonconvex minimization theorem [6]. In [7] Park extended this concept to quasi-metric spaces in order to generalize and unify different versions of Ekeland's variational principle. Park's approach was continued by Al-Homidan et al. [8], and recently by Latif and Al-Mezel [9], and Marín et al. $[10,11]$, among others. Thus in [8] were obtained extensions and generalizations of Caristi-Kirk's type fixed point theorem 
[12] as well as a Takahashi type minimization theorem and generalizations of Ekeland's variational principle and of Nadler's fixed point theorem [13], respectively, while in [9-11] were proved several fixed point theorems for single and setvalued maps on quasi-metric spaces by using $Q$-functions in the sense of [8] and $w$-distances.

Definition 2 (see $[7,8]$ ). A $w$-distance for a quasi-metric space $(X, d)$ is a function $q: X \times X \rightarrow \mathbb{R}^{+}$satisfying the following three conditions:

(W1) $q(x, y) \leq q(x, z)+q(z, y)$ for all $x, y, z \in X$;

(W2) $q(x, \cdot): X \rightarrow \mathbb{R}^{+}$is lower semicontinuous on $\left(X, \tau_{d^{-1}}\right)$ for all $x \in X$

(W3) for each $\varepsilon>0$ there exists $\delta>0$ such that $q(x, y) \leq \delta$ and $q(x, z) \leq \delta$, imply $d(y, z) \leq \varepsilon$.

Note that every quasi-metric $d$ on $X$ satisfies conditions (W1) and (W2) above.

If $d$ is a metric on $X$, then Definition 2 provides the notion of a $w$-distance for the metric space $(X, d)$ as defined in [4]. In particular, every metric $d$ on $X$ is a $w$-distance for $(X, d)$.

Unfortunately, the situation is quite different when $d$ is a quasi-metric. In fact, it was shown in [10] that if a quasi-metric $d$ on $X$ is also a $w$-distance for $(X, d)$, then the topology $\tau_{d}$ induced by $d$ is metrizable. Hence, many distinguished examples of nonmetrizable quasi-metrizable topological spaces do not admit any compatible quasi-metric which is also a $w$-distance.

Motivated by this fact, in Section 2 we will show that the use of (pre)ordered quasi-metric spaces, with a suitable adaptation of the notion of $w$-distance to this setting, allows us to generate several interesting examples of preordered quasi-metric spaces $(X, d)$ for which the quasi-metric $d$ is a $w$-distance in this new sense. In Section 3 we will prove a fixed point theorem for set-valued maps on complete preordered quasi-metric spaces by means of the modified notion of $w$ distance, that generalizes and extends several well-known fixed point theorems and allows us to deduce fixed point results involving the lower Hausdorff distance of a complete preordered quasi-metric space. We illustrate these results with some examples.

\section{Preordered Quasi-Metric Spaces, $w_{\leq}$-Distances, and Examples}

We start this section by recalling some pertinent concepts.

A preorder on a (nonempty) set $X$ is a reflexive and transitive (binary) relation $\preceq$ on $X$. If, in addition, $\preceq$ is antisymmetric (i.e., condition $x \preceq y$ and $y \preceq x$, implies $x=y), \preceq$ is called a partial order or, simply, an order on $X$. The usual order on $\mathbb{R}$ is denoted by $\leq$.

Let $\preceq$ be a preorder on $X$. Given $x \in X$ the set $\{y \in X$ : $x \preceq y\}$ will be denoted by $\uparrow\{x\}$. A sequence $\left(x_{n}\right)_{n \in \omega}$ in $X$ is said to be nondecreasing if $x_{n} \preceq x_{n+1}$ for all $n \in \omega$.

Remark 3. Given a (nonempty) set $X$, the (trivial) relation $\preceq^{t}$ given by $x \leq^{t} y$ if and only if $x, y \in X$ is obviously a preorder on $X$.
According to [14], a (pre)ordered quasi-metric space is a triple $(X, \preceq, d)$ such that $\preceq$ is a (pre)order on $X$ and $d$ is a quasi-metric on $X$.

Observe that if $(X, d)$ is a quasi-metric space, then the relation $\leq_{d}$ on $X$ defined by $x \leq_{d} y$ if and only if $d(x, y)=0$ is a partial order on $X$ called the specialization order of $(X, d)$. So $\left(X, \leq_{d}, d\right)$ is an ordered quasi-metric space.

Definition 4. A $w_{\leq}$-distance for a preordered quasi-metric space $(X, \preceq, d)$ is a function $q: X \times X \rightarrow \mathbb{R}^{+}$satisfying conditions (W1) and (W2) of Definition 2, and: (W $\left.\mathrm{W}_{\leq} 3\right)$ for each $\varepsilon>0$ there exists $\delta>0$ such that $q(x, y) \leq \delta, q(x, z) \leq \delta$, and $y \preceq z$, imply $d(y, z) \leq \varepsilon$.

Example 5. Let $q$ be a $w$-distance for a quasi-metric space $(X, d)$. Then $q$ is obviously a $w_{\preceq}$-distance for the preordered quasi-metric space $\left(X, \preceq^{t}, d\right)$.

Example 6. Let $(X, d)$ be a quasi-metric space. Consider the ordered quasi-metric space $\left(X, \leq_{d}, d\right)$. Of course, $d$ satisfies conditions (W1) and (W2). Moreover, it trivially satisfies condition $\left(\mathrm{W}_{\preceq} 3\right)$ of Definition 4 . Hence $d$ is a $w_{\preceq}$-distance for $\left(X, \leq_{d}, d\right)$.

Example 7. Let $X=\mathbb{R}$ and let $d_{S}$ be the quasi-metric on $X$ given by $d_{S}(x, y)=y-x$ if $x \leq y$, and $d_{S}(x, y)=1$ if $x>y$. Then $d_{S}$ induces the Sorgenfrey topology on $X$. We show that $d_{S}$ is is a $w_{\leq}$-distance for the ordered $T_{1}$ quasi-metric $(X, \leq$ ,$\left.d_{S}\right)$. Indeed, since $d_{S}$ is a quasi-metric, we only need to show condition $\left(\mathrm{W}_{\prec} 3\right)$ of Definition 4 . To this end, choose $\varepsilon>0$. Put $\delta=\min \{1 / 2, \varepsilon\}$, and let $d_{S}(x, y) \leq \delta$ and $d_{S}(x, z) \leq \delta$ with $y \leq z$. Therefore $d_{S}(x, y)=y-x \leq \varepsilon$ and $d_{S}(x, z)=$ $z-x \leq \varepsilon$. Since $y \leq z$, we have $d_{S}(y, z)=z-y \leq z-x \leq \varepsilon$. We conclude that $d_{S}$ is a $w_{\leq}$-distance for $\left(X, \leq, d_{S}\right)$.

Our next example should be compared with Example 3.1 of [8]. Recall $[3,15]$ that an asymmetric norm on a real vector space $X$ is a function $p: X \rightarrow \mathbb{R}^{+}$such that for each $x, y \in X$ and $r \in \mathbb{R}^{+}$: (i) $p(x)=p(-x)=0$ if and only if $x=0$; (ii) $p(r x)=r p(x)$; (iii) $p(x+y) \leq p(x)+p(y)$.

Then, the pair $(X, p)$ is called an aysmmetric normed space. Asymmetric norms are called quasi-norms in [16, 17], and so forth.

Example 8. Let $(X, \preceq,\|\cdot\|)$ be a normed lattice. Denote by $X^{+}$the positive cone of $X$, that is, $X^{+}:=\{x \in X: 0 \preceq x\}$, and define $\|\cdot\|^{+}: X \rightarrow \mathbb{R}^{+}$as $\|x\|^{+}=\|x \vee \mathbf{0}\|$ for all $x \in X$. Then $\|\cdot\|^{+}$is an aysmmetric norm on $X$ (see, e.g., [17, Theorem 3.1]), and thus the function $d$ defined by $d(x, y)=$ $\|y-x\|^{+}$for all $x, y \in X$ is a quasi-metric on $X$, so $(X, \preceq, d)$ is an ordered quasi-metric space. Hence $\left(X^{+}, \preceq, d_{+}\right)$is also an ordered quasi-metric space, where $d_{+}$denotes the restriction of $d$ to $X^{+}$.

We will show that the function $q$ defined by $q(x, y)=\|y\|$ for all $x, y \in X^{+}$, is a $w_{\leq}$-distance for $\left(X^{+}, \preceq, d_{+}\right)$. Indeed, first note that condition (W1) is trivially satisfied. Now fix $x \in X^{+}$ and let $\left(y_{n}\right)_{n \in \omega}$ be a sequence in $X^{+}$such that $\lim d_{+}\left(y_{n}, y\right)=$ 0 for some $y \in X^{+}$. Since

$$
\begin{aligned}
q(x, y) & =\|y\|=\|y\|^{+}=\left\|y-y_{n}+y_{n}\right\|^{+} \\
& \leq\left\|y-y_{n}\right\|^{+}+\left\|y_{n}\right\|^{+}=d_{+}\left(y_{n}, y\right)+q\left(x, y_{n}\right)
\end{aligned}
$$


for all $n \in \omega$, we deduce that $q(x, \cdot)$ is lower semicontinuous for $\left(X^{+}, \tau_{\left(d_{+}\right)^{-1}}\right)$, and thus condition (W2) is satisfied. Finally, choose $\varepsilon>0$ and put $\delta=\varepsilon / 2$. Suppose $q(x, y) \leq \delta$ and $q(x, z) \leq \delta$ with $y \preceq z$. Therefore

$$
\begin{aligned}
d_{+}(y, z) & =\|z-y\|^{+}=\|(z-y) \vee 0\|=\|z-y\| \\
& \leq\|z\|+\|y\|=q(x, z)+q(x, y) \leq 2 \delta=\varepsilon .
\end{aligned}
$$

Consequently condition $\left(\mathrm{W}_{\preceq} 3\right)$ is also satisfied, so $q$ is a $w_{\varsigma^{-}}$ distance for $\left(X^{+}, \preceq, d_{+}\right)$.

Definition 9. A preordered quasi-metric space $(X, \preceq, d)$ is called complete if for each nondecreasing Cauchy sequence $\left(x_{n}\right)_{n \in \omega}$ the following two conditions hold:

$\left(\mathrm{i}_{1}\right)$ there exists $z \in X$ satisfying $\lim _{n} d\left(x_{n}, z\right)=0$;

$\left(\mathrm{i}_{2}\right)$ each $z \in X$ satisfying $\lim _{n} d\left(x_{n}, z\right)=0$ verifies that $x_{n} \preceq z$ for all $n \in \omega$.

Next we give some examples of complete preordered quasi-metric spaces.

Example 10. Let $(X, d)$ be any complete quasi-metric space. Then $\left(X, \preceq^{t}, d\right)$ is obviously a complete preordered quasimetric space.

Example 11. Let $\preceq$ be a partial order on a set $X$. Then, for every complete quasi-metric $d$ on $X$ such that $d(x, y)=0$ if and only if $x \preceq y$, we have that $(X, \preceq, d)$ is a complete ordered quasi-metric space (note that in this case the partial order $\preceq$ coincides with the specialization order $\leq_{d}$ ). Indeed, let $\left(x_{n}\right)_{n \in \omega}$ be a nondecreasing Cauchy sequence and let $z \in X$ be such that $\lim _{n} d\left(x_{n}, z\right)=0$. Choose any $n \in \omega$. Then, for each arbitrary $\varepsilon>0$ there is $m>n$ such that $d\left(x_{m}, z\right)<\varepsilon$. Since $x_{n} \preceq x_{m}$, we have $d\left(x_{n}, x_{m}\right)=0$, so by the triangle inequality, $d\left(x_{n}, z\right)<\varepsilon$. Since $\varepsilon$ is arbitrary we deduce that $d\left(x_{n}, z\right)=0$. Hence $x_{n} \preceq z$.

Example 12. Let $\left(X, \leq, d_{S}\right)$ be the ordered $T_{1}$ quasi-metric space of Example 7. If $\left(x_{n}\right)_{n \in \omega}$ is a Cauchy sequence in $\left(X, d_{S}\right)$ that is also nondecreasing, then it is clear that $\lim _{n} d_{S}\left(x_{n}, z\right)=$ 0 only for $z=\sup \left\{x_{n}: n \in \omega\right\}$. Therefore $\left(X, \leq, d_{S}\right)$ is a complete ordered $T_{1}$ quasi-metric space.

Example 13. Let $X=\mathbb{R}^{+}$and let $d$ be the complete quasimetric on $X$ given by $d(x, y)=\max \{y-x, 0\}$ for all $x, y \in$ $X$. Then $(X, \leq, d)$ is not a complete preordered quasi-metric space in our sense because any (nondecreasing Cauchy) sequence $\left(x_{n}\right)_{n \in \omega}$ in $X$ satisfies $\lim _{n} d\left(x_{n}, 0\right)=0$, so condition $\left(\mathrm{i}_{2}\right)$ of Definition 9 does not hold. However, since $d(x, y)=0$ if and only if $x \geq y$, it follows from Example 11 that $(X, \geq, d)$ is a complete ordered quasi-metric space.

\section{Fixed Point Results}

Answering a question posed by Reich [18], Mizoguchi and Takahashi [19] (see also [20, 21]) obtained a set-valued generalization-improvement of the Rakotch fixed point theorem [22, Corollary of Theorem 2]. Recently, Latif and
Al-Mezel [9, Theorem 2.3] extended Mizoguchi-Takahashi's theorem to the framework of complete $T_{1}$ quasi-metric spaces by using $w$-distances (actually they states their result in a slightly more general form by using $Q$-functions in the sense of [8], instead of $w$-distances). Here we obtain a fixed point theorem for complete preordered quasi-metric spaces from which [9, Theorem 2.3] can be deduced as a special case. Several other consequences are deduced and some illustrative examples are given.

We first introduce the notions of contractiveness that we will use in the rest of the paper.

If $(X, d)$ is a quasi-metric space, we denote by $2^{X}$ the set of all nonempty subsets of $X$ and by $C_{d}(X)$ the set of all nonempty $\tau_{d}$-closed subsets of $X$.

Definition 14. Let $(X, \preceq, d)$ be a preordered quasi-metric space and let $T: X \rightarrow 2^{X}$ be a set-valued map such that $T x \cap \uparrow\{x\} \neq \emptyset$ for all $x \in X$. We say that $T$ is $w_{\leq^{-}}$ contractive if there exist a $w_{\leq}$-distance $q$ for $(X, \preceq, d)$ and a constant $r \in(0,1)$, such that for each $x, y \in X$, with $x \preceq y$, and $u \in T x \cap \uparrow\{x\}$ there is $v \in T y \cap \uparrow\{y\}$ satisfying $q(u, v) \leq r q(x, y)$.

Definition 15. Let $(X, \preceq, d)$ be a preordered quasi-metric space and let $T: X \rightarrow 2^{X}$ be a set-valued map such that $T x \cap \uparrow\{x\} \neq \emptyset$ for all $x \in X$. We say that $T$ is generalized $w_{\leq}$-contractive if there exist a $w_{\leq}$-distance $q$ for $(X, \preceq, d)$ and a function $\alpha: \mathbb{R}^{+} \rightarrow[0,1)$ with $\limsup _{r \rightarrow t^{+}} \alpha(r)<1$ for all $t \in \mathbb{R}^{+}$, and such that for each $x, y \in X$, with $x \preceq y$, and $u \in T x \cap \uparrow\{x\}$ there is $v \in T y \cap \uparrow\{y\}$ satisfying $q(u, v) \leq \alpha(q(x, y)) q(x, y)$.

Theorem 16. Let $(X, \preceq, d)$ be a complete preordered quasimetric space and $T: X \rightarrow C_{d}(X)$ be a generalized $w_{\varsigma^{-}}$ contractive set-valued map. Then $T$ has a fixed point.

Proof. Since $T$ is generalized $w_{\leq}$-contractive, there is a $w_{\leq^{-}}$ distance $q$ for $(X, \preceq, d)$ and a function $\alpha: \mathbb{R}^{+} \rightarrow[0,1)$ with $\limsup _{r \rightarrow t^{+}} \alpha(r)<1$ for all $t \in \mathbb{R}^{+}$, and such that for each $x, y \in X$, with $x \preceq y$, and $u \in T x \cap \uparrow\{x\}$ there is $v \in T y \cap \uparrow$ $\{y\}$ satisfying

$$
q(u, v) \leq \alpha(q(x, y)) q(x, y) .
$$

Fix $x_{0} \in X$. Since $T x_{0} \cap \uparrow\left\{x_{0}\right\} \neq \emptyset$ there exists $x_{1} \in T x_{0}$ such that $x_{0} \preceq x_{1}$. Taking $x=x_{0}$ and $y=u=x_{1}$, we deduce the existence of an $x_{2} \in T x_{1}$ such that $x_{1} \preceq x_{2}$ and

$$
q\left(x_{1}, x_{2}\right) \leq \alpha\left(q\left(x_{0}, x_{1}\right)\right) q\left(x_{0}, x_{1}\right) .
$$

Repeating the above argument, there is $x_{3} \in T x_{2}$ such that $x_{2} \preceq x_{3}$ and

$$
q\left(x_{2}, x_{3}\right) \leq \alpha\left(q\left(x_{1}, x_{2}\right)\right) q\left(x_{1}, x_{2}\right) .
$$

Hence, following this process we construct a sequence $\left(x_{n}\right)_{n \in \omega}$ in $X$ such that for every $n \in \mathbb{N}$,
(a) $x_{n+1} \in T x_{n}$,
(b) $x_{n} \preceq x_{n+1}$, and
(c) $q\left(x_{n}, x_{n+1}\right) \leq \alpha\left(q\left(x_{n-1}, x_{n}\right)\right) q\left(x_{n-1}, x_{n}\right)$. 
Next we show that $\left(x_{n}\right)_{n \in \omega}$ is a Cauchy sequence in the quasi-metric space $(X, d)$.

To this end, first suppose that there is $k \in \omega$ such that $q\left(x_{k}, x_{k+1}\right)=0$. Thus $q\left(x_{n}, x_{m}\right)=0$ whenever $k<n<m$, by conditions (c) and (W1). Then, from conditions (b) and $\left(\mathrm{W}_{\preceq} 3\right)$ we deduce that $\left(x_{n}\right)_{n \in \omega}$ is a Cauchy sequence in $(X, d)$.

Now suppose that $q\left(x_{n}, x_{n+1}\right)>0$ for all $n \in \omega$. Put $r_{n}=q\left(x_{n}, x_{n+1}\right), n \in \omega$. Then $\left(r_{n}\right)_{n \in \omega}$ is a strictly decreasing sequence of non-negative real numbers. Let $c \in \mathbb{R}^{+}$be such that $\lim _{n} r_{n}=c$. Then

$$
\lim \sup _{r_{n} \rightarrow c} \alpha\left(r_{n}\right)<1 .
$$

Hence there exist $b \in(0,1)$ and $n_{0} \in \mathbb{N}$ such that $\alpha\left(r_{n}\right)<b$ for all $n \geq n_{0}$. By condition (c) we deduce that

$$
\begin{aligned}
q\left(x_{n}, x_{n+1}\right) & <b q\left(x_{n-1}, x_{n}\right)<b^{2} q\left(x_{n-2}, x_{n-1}\right) \\
& <\cdots<b^{n-n_{0}} q\left(x_{n_{0}}, x_{n_{0}+1}\right)
\end{aligned}
$$

for all $n>n_{0}$. Now choose $\varepsilon>0$. Then, there is $\delta>0$ for which condition $\left(\mathrm{W}_{\preceq} 3\right)$ follows. Since by $(7)$ and (W1) there is $n_{1} \in \mathbb{N}$ such that $q\left(x_{n}, x_{m}\right) \leq \delta$ whenever $n_{1} \leq n<m$, we deduce from $\left(\mathrm{W}_{\preceq} 3\right)$ that $d\left(x_{n}, x_{m}\right) \leq \varepsilon$ whenever $n_{1}<n<$ $m$. Therefore $\left(x_{n}\right)_{n \in \omega}$ is a nondecreasing Cauchy sequence in $(X, \preceq, d)$.

Since $(X, \preceq, d)$ is a complete preordered quasi-metric space, there exists $z \in X$ such that $\lim _{n} d\left(x_{n}, z\right)=0$ and $x_{n} \preceq z$ for all $n \in \omega$.

Next we show that $\lim _{n} q\left(x_{n}, z\right)=0$.

Indeed, choose $\varepsilon>0$. Then, there is $n_{0} \in \mathbb{N}$ such that $q\left(x_{n}, x_{m}\right)<\varepsilon / 2$ whenever $n_{0} \leq n<m$. Given $n \geq n_{0}$ there is, by condition (W2), an $n_{1}>n$ such that

$$
q\left(x_{n}, z\right)-q\left(x_{n}, x_{n_{1}}\right)<\frac{\varepsilon}{2} \text {. }
$$

Thus

$$
q\left(x_{n}, z\right)<\frac{\varepsilon}{2}+q\left(x_{n}, x_{n_{1}}\right)<\varepsilon .
$$

Therefore $\lim _{n} q\left(x_{n}, z\right)=0$.

Finally, since $x_{n} \preceq z$ for all $n \in \omega$, we can find a sequence $\left(v_{n}\right)_{n \in \mathbb{N}}$ in $T z$ such that $z \preceq v_{n}$ and

$$
q\left(x_{n}, v_{n}\right) \leq \alpha\left(q\left(x_{n-1}, z\right)\right) q\left(x_{n-1}, z\right)
$$

for all $n \in \mathbb{N}$. Hence $\lim _{n} q\left(x_{n}, v_{n}\right)=0$. We deduce from $\left(\mathrm{W}_{\preceq} 3\right)$ that $\lim _{n} d\left(z, v_{n}\right)=0$. So $z \in T z$ because $T z \in C_{d}(X)$. This concludes the proof.

Corollary 17. Let $(X, \preceq, d)$ be a complete preordered quasimetric space and $T: X \rightarrow C_{d}(X)$ be a $w_{\leq}$-contractive setvalued map. Then $T$ has a fixed point.

Corollary 18. Let $(X, \preceq, d)$ be a complete preordered $T_{1}$ quasimetric space for which $d$ is a $w_{\leq}$-distance and let $T: X \rightarrow X$ be a self-map. If there is a function $\alpha: \mathbb{R}^{+} \rightarrow[0,1)$ with $\lim \sup _{r \rightarrow t^{+}} \alpha(r)<1$ for all $t \in \mathbb{R}^{+}$, and such that for each $x, y \in X$, with $x \preceq y$, one has

$$
d(T x, T y) \leq \alpha(d(x, y)) d(x, y),
$$

then $T$ has a fixed point.
Proof. Since $\tau_{d}$ is a $T_{1}$ topology, then $T x \in C_{d}(X)$ for all $x \in X$. The result is now an immediate consequence of Theorem 16.

Remark 19. Putting $\preceq=\preceq^{t}$ and taking into account Example 5, we deduce that [9, Theorem 2.3] and [8, Theorem 6.1] are, for $w$-distances, special cases of Theorem 16 and Corollary 17 respectively, whereas Corollary 18 provides a quasi-metric generalization of Rakotch's fixed point theorem.

Next we give an easy example where Corollary 17, and hence Theorem 16, can be applied to the involved complete ordered $T_{1}$ quasi-metric space $(X, \preceq, d)$, but not to the complete ordered metric space $\left(X, \preceq, d^{s}\right)$.

Example 20. Let $\left(X, \leq d_{S}\right)$ be the complete ordered $T_{1}$ quasimetric space of Example 12 and let $T: X \rightarrow X$ defined by $T x=x / 2$ for all $x \in X$. Since $d$ is a $w_{\leq}$-distance for $\left(X, \leq, d_{S}\right)$ (see Example 7), and for each $x, y \in X$ with $x \leq y$, we have

$$
d_{S}(T x, T y)=\frac{y-x}{2}=\frac{1}{2} d_{S}(x, y)
$$

then all conditions of Corollary 17, and thus of Theorem 16, are satisfied. However, for $x, y \in X$ with $0 \leq x<y \leq 1$, we have

$$
\left(d_{S}\right)^{s}(T x, T y)=1=\left(d_{S}\right)^{s}(x, y) \text {, }
$$

so Theorem 16 cannot be applied to the complete ordered metric space $\left(X, \leq,\left(d_{S}\right)^{s}\right)$ and the self-map $T$.

In the sequel we will apply Corollary 17 to deduce a fixed point result for set-valued maps on complete preordered $T_{1}$ quasi-metric spaces involving the (lower) Hausdorff distance.

Let $(X, d)$ be a quasi-metric space. For each $A, B \in C_{d}(X)$ let

$$
\begin{gathered}
H_{d}^{-}(A, B)=\sup _{a \in A} d(a, B), \quad H_{d}^{+}(A, B)=\sup _{b \in B} d(A, b), \\
H_{d}(A, B)=\max \left\{H_{d}^{-}(A, B), H_{d}^{+}(A, B)\right\} .
\end{gathered}
$$

Then $H_{d}^{-}, H_{d}^{+}$and $H_{d}$ will be called the lower Hausdorff distance of $(X, d)$, the upper Hausdorff distance of $(X, d)$ and the Hausdorff distance of $(X, d)$, respectively (compare e.g., [23-26]).

It is interesting to note that $H_{d}^{-}, H_{d}^{+}$and $H_{d}$ are extended quasi-pseudometrics on $C_{d}(X)$, but not quasi-metrics, in general.

Corollary 21. Let $(X, \preceq, d)$ be a complete preordered $T_{1}$ quasimetric space for which $d$ is a $w_{\leq}$-distance and let $T: X \rightarrow$ $C_{d}(X)$ be a set-valued map such that $T x \cap \uparrow\{x\} \neq \emptyset$ for all $x \in X$. If there is $r \in(0,1)$ such that for each $x, y \in X$, with $x \preceq y$,

$$
H_{d}^{-}(T x \cap \uparrow\{x\}, T y \cap \uparrow\{y\}) \leq r d(x, y),
$$

then $T$ has a fixed point. 
Proof. Take $s \in(r, 1)$. Then $T$ is a $w_{\leq}$-contractive set-valued map for the $w_{\leq}$-distance $d$ and the constant $s$. By Corollary 17 , $T$ has a fixed point.

Remark 22. Observe that for the ordered quasi-metric space $\left(X, \leq_{d}, d\right)$, any set-valued map $T: X \rightarrow C_{d}(X)$ such that $T x \cap \uparrow\{x\} \neq \emptyset$ for all $x \in X$, satisfies that every $x \in X$ is a fixed point of $T$. Indeed, condition $T x \cap \uparrow\{x\} \neq \emptyset$ implies $T x \cap \mathrm{cl}_{\tau_{d^{-1}}}\{x\} \neq \emptyset$, so $x \in \mathrm{cl}_{\tau_{d}} T x$, that is, $x \in T x$. Note also that the contraction condition (15) is, in this case, equivalent to the following:

$$
d(x, y)=0 \Longrightarrow H_{d}^{-}\left(T x \cap \mathrm{cl}_{\tau_{d^{-1}}}\{x\}, T y \cap \mathrm{cl}_{\tau_{d^{-1}}}\{y\}\right)=0 .
$$

We finish the paper with two examples that illustrate Corollary 21 and Remark 22, respectively.

Example 23. Let $X$ be the set of all continuous functions from $[0,1]$ into itself and let $d$ be the $T_{1}$ quasi-metric on $X$ defined as (compare [27, Example 4]):

$$
\begin{gathered}
d(f, g)=\sup \{g(x)-f(x): x \in[0,1]\} \\
\quad \text { if } f(x) \leq g(x) \forall x \in[0,1], \\
d(f, g)=1, \quad \text { otherwise. }
\end{gathered}
$$

Let $\preceq$ be the usual pointwise partial order on $X$, that is, $f \preceq g$ if and only if $f(x) \leq g(x)$ for all $x \in[0,1]$. By standard arguments we deduce that $(X, \preceq, d)$ is a complete ordered $T_{1}$ quasi-metric space: Indeed, given a nondecreasing Cauchy sequence $\left(f_{n}\right)_{n \in \omega}$ in $(X, \preceq, d)$, then $\lim _{n} d\left(f_{n}, f\right)=0$ only for the function $f \in X$ defined by $f(x)=\sup \left\{f_{n}(x): n \in \omega\right\}$ for all $x \in[0,1]$.

Moreover $d$ is a $w_{\leq}$-distance for $(X, \preceq, d)$ because given $\varepsilon>0$ we take $\delta=\min \{1 / 2, \varepsilon\}$, and then for $d(f, g) \leq \delta$, $d(f, h) \leq \delta$ and $g \preceq h$, we obtain

$$
\begin{aligned}
d(g, h) & =\sup \{h(x)-g(x): x \in[0,1]\} \\
& \leq \sup \{h(x)-f(x): x \in[0,1]\}=d(f, h) \leq \delta \leq \varepsilon .
\end{aligned}
$$
by

Now construct the set-valued map $T: X \rightarrow C_{d}(X)$ given

$$
\begin{aligned}
T f=\left\{f_{n}\right. & \in X: f_{n}(x) \\
& \left.=\frac{f(x)+2 n-1}{2 n} \forall x \in[0,1], n \in \mathbb{N}\right\} .
\end{aligned}
$$

Note that $T f \in C_{d}(X)$. Indeed, suppose that there is $h \in$ $\mathrm{cl}_{d} T f \backslash T f$. Then, there is a subsequence $\left(f_{n_{k}}\right)_{k \in \omega}$ of $\left(f_{n}\right)_{n \in \omega}$ such that $d\left(h, f_{n_{k}}\right)<2^{-k}$ for all $k \in \omega$. Since $f_{n} \preceq f_{n+1}$ for all $n$, we can assume, without loss of generality, that $f_{n_{k}} \preceq f_{n_{k+1}}$ for all $k$. Consequently, we have for each $x \in[0,1]$ and each $k$,

$$
f_{n_{0}}(x) \leq f_{n_{k}}(x)<h(x)+2^{-k}
$$

Since $h(x) \leq f_{n_{0}}(x)$, we deduce that $f_{n_{0}}(x)=h(x)$ for all $x \in[0,1]$, which contradicts that $h \notin T f$. We conclude that $T f \in C_{d}(X)$.

Moreover $T f \cap \uparrow\{f\} \neq \emptyset$ for all $f \in X$ because $f \preceq f_{n}$ for all $n \in \mathbb{N}$ and thus $T f \cap \uparrow\{f\}=T f$.

Finally, let $f, g \in X$, with $f \preceq g$, and $u \in T f \cap \uparrow\{f\}$. Then, there is $n \in \mathbb{N}$ such that $u(x)=(f(x)+2 n-1) / 2 n$ for all $x \in[0,1]$. Taking $v(x)=(g(x)+2 n-1) / 2 n$ for all $x \in[0,1]$, we have $v \in \operatorname{Tg} \cap \uparrow\{g\}, u \preceq v$, and

$$
d(u, v)=\sup \left\{\frac{g(x)-f(x)}{2 n}: x \in[0,1]\right\} \leq \frac{1}{2} d(f, g) \text {. }
$$

Hence

$$
H_{d}^{-}(T f \cap \uparrow\{f\}, T g \cap \uparrow\{g\}) \leq \frac{1}{2} d(f, g) .
$$

By Corollary 21, $T$ has a fixed point. In fact, the function $h$ defined by $h(x)=1$ for all $x \in[0,1]$, satisfies $h \in T h$.

Example 24. Consider the Banach lattice $\left(l_{1}, \preceq,\|\cdot\|\right)$, where $l_{1}$ denotes the vector space of all infinite sequences $\mathbf{x}:=\left(x_{n}\right)_{n \in \omega}$ of real numbers such that $\sum_{n=0}^{\infty}\left|x_{n}\right|<\infty, \preceq$ denotes the usual order on $l_{1}$ and $\|\mathbf{x}\|:=\sum_{n=0}^{\infty}\left|x_{n}\right|$ for all $\mathbf{x}:=\left(x_{n}\right)_{n \in \omega} \in l_{1}$.

Now denote by $l_{1}^{+}$the positive cone of $l_{1}$ and by $d_{+}$the quasi-metric on $l_{1}^{+}$defined by $d_{+}(\mathbf{x}, \mathbf{y})=\|(\mathbf{y}-\mathbf{x}) \vee \mathbf{0}\|$ for all $\mathbf{x}, \mathbf{y} \in l_{1}^{+}$(compare Example 8 ). Then $\left(l_{1}^{+}, d_{+}\right)$is a complete quasi-metric space by [28, Theorem 2].

Let $\psi: l_{1}^{+} \rightarrow l_{1}^{+}$be nondecreasing and such that $\psi(\mathbf{x}) \preceq \mathbf{x}$ for all $\mathbf{x} \in l_{1}^{+}$. Define $T: l_{1}^{+} \rightarrow C_{d_{+}}\left(l_{1}^{+}\right)$as

$$
T \mathbf{x}=\left\{\mathbf{y} \in l_{1}^{+}: \psi(\mathbf{x}) \preceq \mathbf{y}\right\}
$$

for all $\mathbf{x} \in l_{1}^{+}$. Then $T \mathbf{x} \cap \mathrm{cl}_{\tau_{d^{-1}}}\{\mathbf{x}\}=\left\{\mathbf{y} \in l_{1}^{+}: \psi(\mathbf{x}) \preceq \mathbf{y} \preceq \mathbf{x}\right\}$ (compare Remark 22). In fact $\mathbf{x} \in T \mathbf{x}$ for all $\mathbf{x} \in l_{1}^{+}$.

Finally note that given $\mathbf{x}, \mathbf{y} \in l_{1}^{+}$with $d_{+}(\mathbf{x}, \mathbf{y})=0$ and $\mathbf{u} \in T \mathbf{x} \cap \mathrm{cl}_{\tau_{d^{-1}}}\{\mathbf{x}\}$, we have that $\mathbf{y} \preceq \mathbf{x}$ and $\psi(\mathbf{x}) \preceq \mathbf{u} \preceq \mathbf{x}$, so $\psi(\mathbf{y}) \preceq \psi(\mathbf{x}) \preceq \mathbf{u}$, and hence

$$
d_{+}(\mathbf{u}, \psi(\mathbf{y}))=0 .
$$

Since $\psi(\mathbf{y}) \in T \mathbf{y} \cap \mathrm{cl}_{\tau_{d^{-1}}}\{\mathbf{y}\}$, we deduce that condition (16) of Remark 22 is also satisfied.

\section{Acknowledgments}

The authors thank the referees for some useful suggestions and corrections. This research is supported by the Ministry of Economy and Competitiveness of Spain, Grant MTM201237894-C02-01. S. Romaguera and P. Tirado also acknowledge the support of Universitat Politècnica de València, Grant PAID-06-12.

\section{References}

[1] P. Fletcher and W. F. Lindgren, Quasi-Uniform Spaces, Marcel Dekker, New York, NY, USA, 1982. 
[2] H. P. A. Künzi, "Nonsymmetric distances and their associated topologies: about the origins of basic ideas in the area of asymmetric topology," in Handbook of the History of General Topology, C. E. Aull and R. Lowen, Eds., vol. 3, pp. 853-968, Kluwer Academic, Dodrecht, The Netherlands, 2001.

[3] S. Cobzas, Functional Analysis in Asymmetric Normed Spaces, Birkhäauser, Boston, Mass, USA, 2012.

[4] O. Kada, T. Suzuki, and W. Takahashi, "Nonconvex minimization theorems and fixed point theorems in complete metric spaces," Mathematica Japonica, vol. 44, no. 2, pp. 381-391, 1996.

[5] I. Ekeland, "Nonconvex minimization problems," Bulletin of the American Mathematical Society, vol. 1, no. 3, pp. 443-474, 1979.

[6] W. Takahashi, "Existence theorems generalizing fixed point theorems for multivalued mappings," in Fixed Point Theory and Applications, M. A. Théra and J. B. Baillon, Eds., vol. 252, pp. 397-406, Longman, Harlow, UK, 1991.

[7] S. Park, "On generalizations of the Ekeland-type variational principles," Nonlinear Analysis: Theory, Methods \& Applications, vol. 39, pp. 881-889, 2000.

[8] S. Al-Homidan, Q. H. Ansari, and J.-C. Yao, "Some generalizations of Ekeland-type variational principle with applications to equilibrium problems and fixed point theory," Nonlinear Analysis: Theory, Methods \& Applications, vol. 69, no. 1, pp. 126139, 2008.

[9] A. Latif and S. A. Al-Mezel, "Fixed point results in quasimetric spaces," Fixed Point Theory and Applications, vol. 2011, Article ID 178306, 8 pages, 2011.

[10] J. Marín, S. Romaguera, and P. Tirado, "Q-functions on quasimetric spaces and fixed points for multivalued maps," Fixed Point Theory and Applications, vol. 2011, Article ID 603861, 10 pages, 2011.

[11] J. Marín, S. Romaguera, and P. Tirado, "Weakly contractive multivalued maps and w-distances on complete quasi-metric spaces," Fixed Point Theory and Applications, vol. 2011, no. 1, pp. $1-9,2011$.

[12] J. Caristi and W. A. Kirk, "Geometric fixed point theory and inwardness conditions," in The Geometry of Metric and Linear Spaces, vol. 490 of Lecture Notes in Mathematics, pp. 74-83, Springer, Berlin, Germany, 1975.

[13] S. B. Nadler Jr., "Multi-valued contraction mappings," Pacific Journal of Mathematics, vol. 30, pp. 475-488, 1969.

[14] S. Romaguera, "A common fixed point theorem for weakly compatible self-maps on complete preordered quasi-metric spaces," in Weakly Commuting Mappings after Thirty Years in Metric Fixed Point Theory, S. Sessa, Ed., pp. 55-70, Aracne Editrice S.r.l., Rome, Italy, 2012.

[15] C. Alegre, S. Romaguera, and P. Veeramani, "The uniform boundedness theorem in asymmetric normed spaces," Abstract and Applied Analysis, vol. 2012, Article ID 809626, 8 pages, 2012.

[16] C. Alegre, J. Ferrer, and V. Gregori, "On the Hahn-Banach theorem in certain linear quasi-uniform structures," Acta Mathematica Hungarica, vol. 82, no. 4, pp. 325-330, 1999.

[17] J. Ferrer, V. Gregori, and C. Alegre, "Quasi-uniform structures in linear lattices," The Rocky Mountain Journal of Mathematics, vol. 23, no. 3, pp. 877-884, 1993.

[18] S. Reich, "Some fixed point problems," Atti della Accademia Nazionale dei Lincei, vol. 57, no. 3-4, pp. 194-198, 1974.

[19] N. Mizoguchi and W. Takahashi, "Fixed point theorems for multivalued mappings on complete metric spaces," Journal of Mathematical Analysis and Applications, vol. 141, no. 1, pp. 177$188,1989$.
[20] P. Z. Daffer and H. Kaneko, "Fixed points of generalized contractive multi-valued mappings," Journal of Mathematical Analysis and Applications, vol. 192, no. 2, pp. 655-666, 1995.

[21] C. Yu-Qing, “On a fixed point problem of Reich," Proceedings of the American Mathematical Society, vol. 124, no. 10, pp. 30853088, 1996.

[22] E. Rakotch, "A note on contractive mappings," Proceedings of the American Mathematical Society, vol. 13, pp. 459-465, 1962.

[23] G. Berthiaume, "On quasi-uniformities in hyperspaces," Proceedings of the American Mathematical Society, vol. 66, no. 2, pp. 335-343, 1977.

[24] H. P. A. Künzi and C. Ryser, "The Bourbaki quasi-uniformity," Topology Proceedings, vol. 20, pp. 161-183, 1995.

[25] J. Rodríguez-López and S. Romaguera, "Wijsman and hit-andmiss topologies of quasi-metric spaces," Set-Valued Analysis, vol. 11, no. 4, pp. 323-344, 2003.

[26] J. Rodríguez-López and S. Romaguera, "Hypertopologies and asymmetric topology," in Theory and Applications of Proximity, Nearness and Uniformity, G. di Maio and S. Naimpally, Eds., vol. 22, pp. 317-364, Dipartimento di Matematica Seconda Universitá di Napoli, 2008.

[27] D. Doitchinov, "On completeness in quasi-metric spaces," Topology and its Applications, vol. 30, no. 2, pp. 127-148, 1988.

[28] L. M. García-Raffi, S. Romaguera, and E. A. Sánchez-Pérez, "Sequence spaces and asymmetric norms in the theory of computational complexity," Mathematical and Computer Modelling, vol. 36, no. 1-2, pp. 1-11, 2002. 


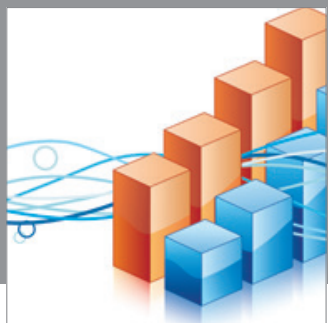

Advances in

Operations Research

mansans

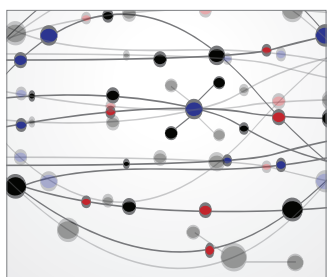

The Scientific World Journal
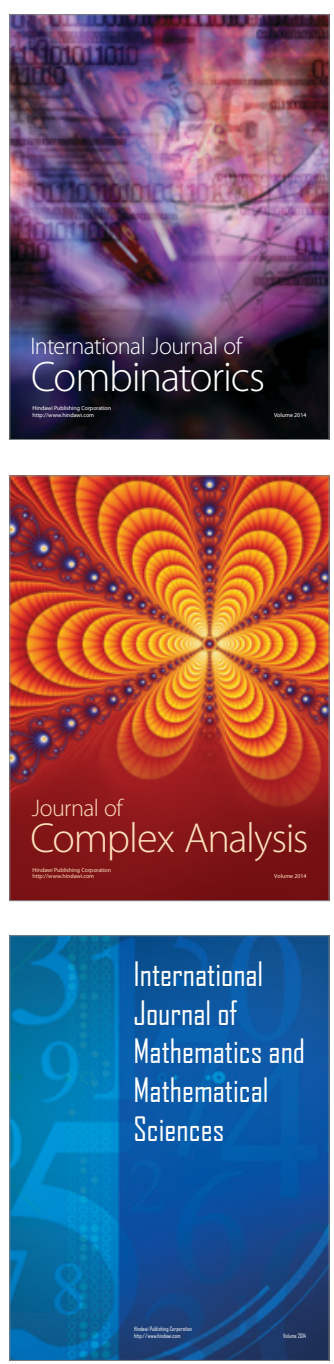
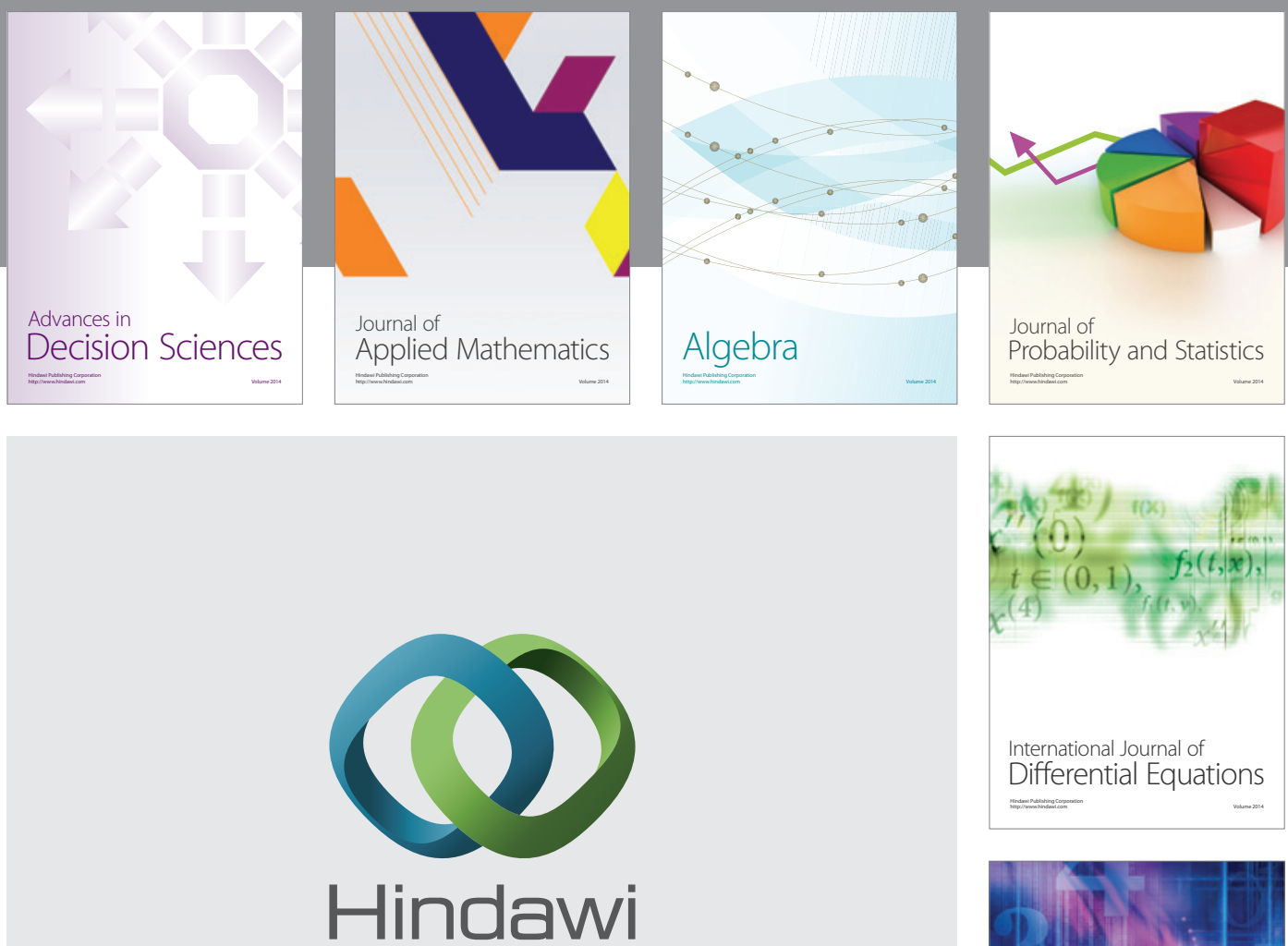

Submit your manuscripts at http://www.hindawi.com
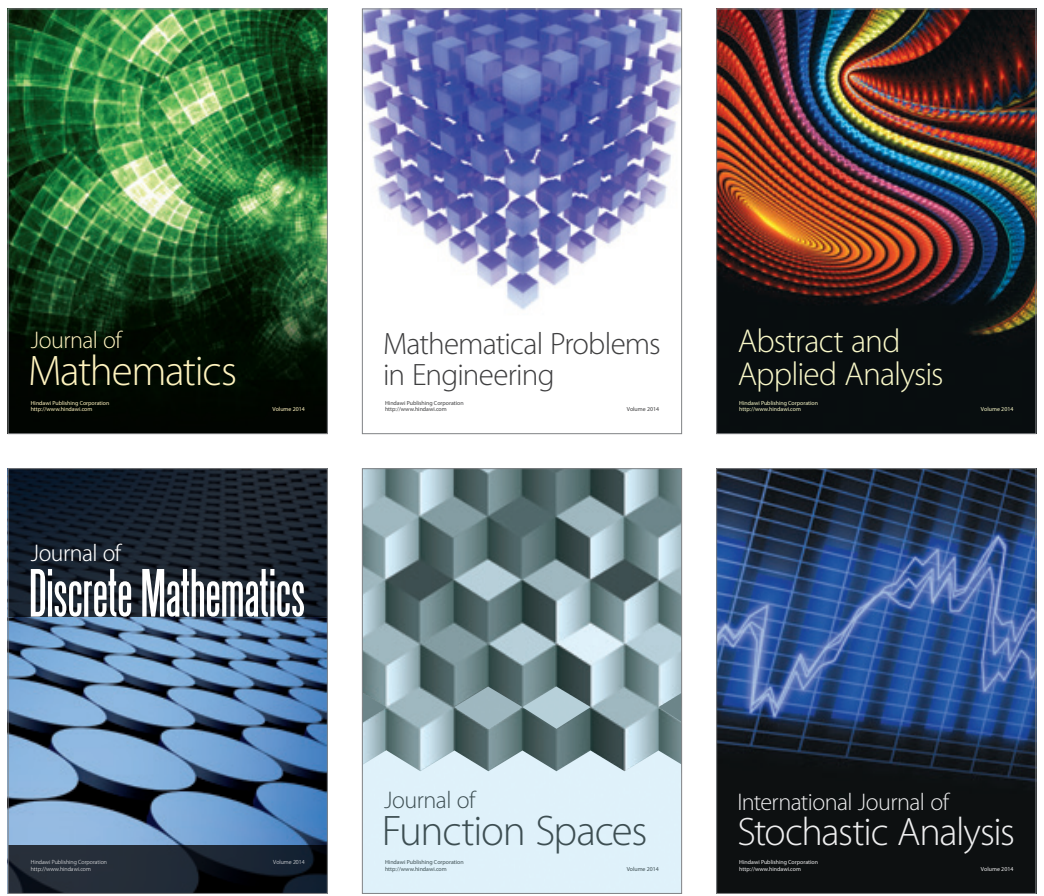

Journal of

Function Spaces

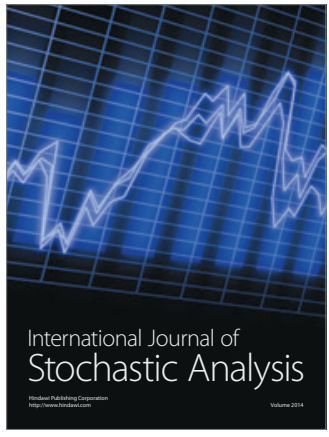

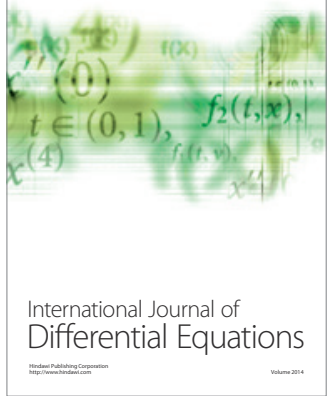
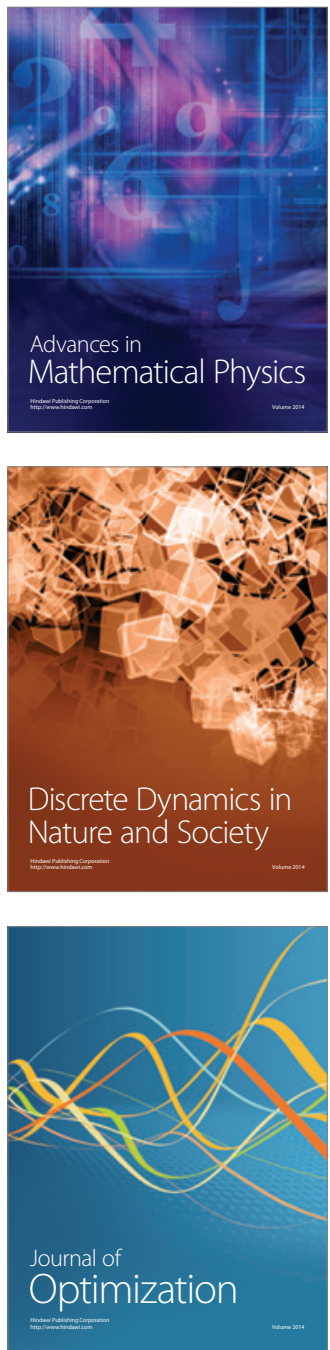